\title{
Short communication: Risk factors for sonographically detectable udder edema in overbagged cows at dairy shows
}

\author{
M. Balmer, ${ }^{1}$ M. Alsaaod, ${ }^{1}$ M. Boesiger, ${ }^{1}$ E. Studer, ${ }^{1}$ R. O'Brien, ${ }^{2}$ G. Schuepbach-Regula, ${ }^{3}$ and A. Steiner ${ }^{1 *}$ \\ ${ }^{1}$ Clinic for Ruminants, Vetsuisse-Faculty, University of Bern, 3001 Bern, Switzerland \\ ${ }^{2}$ BitRads, Nobleboro, ME 04555 \\ ${ }^{3}$ Veterinary Public Health Institute, Vetsuisse-Faculty, University of Bern, 3097 Liebefeld, Switzerland
}

\section{ABSTRACT}

At dairy cow shows, the appearance of the udder is very important. To show cows at their best, udders are often presented in an overbagged state by extending milking intervals before the show. This practice represents an animal welfare issue because it is associated with behavior changes (e.g., excessive abduction and decreased eating time) and may affect the health of the animal. The aim of the current study was to determine the association between various parameters (e.g., milk yield, days in milk, milking procedures, or administration of nonsteroidal anti-inflammatory drugs, steroids, or diuretics) and the sonographically detectable udder edema score. Data were collected from the show catalogs, by interviewing the exhibitors, by analyzing official veterinary treatment protocols and official milking data, via laboratory analysis of blood samples, and by sonographic examinations of the udders at the show. For sonographically detectable udder edema scoring, 3 scans were taken, 1 on each fore quarter and 1 incorporating both rear quarters at the level of the median suspensory ligament. For grading the scans, a scoring system of 4 grades (score $0=$ no edema, $1=$ slight edema, $2=$ moderate edema, 3 = severe edema) was used. Data from 321 cows of different breeds were collected at 4 highly competitive Swiss dairy shows (shows A-D) between January and September 2017. To determine risk factors for sonographically detectable udder edema, data were analyzed with 2 different logistic regression models. In model 1 , the odds ratios were 3.33 (milking intervals of $\geq 16$ h vs. $<14 \mathrm{~h}$ ), 3.84 (show A vs. show $\mathrm{C}$ ), and 7.39 (dairy breeds vs. dual-purpose breeds). Milking intervals of dairy breeds were significantly higher than those of dual-purpose breeds. In model 2 , milking interval was the only significant risk factor with an odds ratio of 9.00 for milking intervals of $\geq 16$

Received June 1, 2018.

Accepted August 29, 2018.

*Corresponding author: adrian.steiner@vetsuisse.unibe.ch h. Only the milking intervals represented a relevant risk factor in both models; therefore, we concluded that the previously described sonographic udder edema scoring is a useful technique for detecting overbagged udders at dairy shows. Its routine implementation may improve cow welfare at dairy shows.

Key words: overbagging, udder edema, sonography, dairy show, welfare

\section{Short Communication}

At dairy cow shows, cows are often presented with an overbagged udder due to "prolonged duration of not milking" (Waller et al., 2007). This practice is common and aims to increase the size of the udder to show the judge the milking potential of a cow. This represents an animal welfare issue because the cows' well-being is disturbed after prolonged milking intervals (PMI; Kohler et al., 2016); changes in behavior and nonphysiological status were identified in cows after PMI. Nonphysiological processes included milk leaking and edema formation. Edema appears sonographically as alternating hyperechoic and hypoechoic parallel lines in the subcutaneous space at defined locations (Waller et al., 2007; O'Brien, 2017). Flunixin meglumine or other nonsteroidal anti-inflammatory drugs (NSAID) or steroidal anti-inflammatory drugs (SAID), which may reduce edema formation according to their antiinflammatory effect, are routinely given at shows to decrease pain associated with PMI. Current techniques used to determine udder filling are not appropriate for show cows because they are not specific and do not allow the identification of a threshold value. Recently, a sonographic udder edema scoring system, allowing objective determination of the severity of udder edema in show cows after a PMI, was described and evaluated (Balmer et al., 2018). The authors found higher inter- and intraobserver reliability of sonographic udder edema scoring compared with visual scoring. Therefore, udder edema score determined sonographically was found to be more suitable than the visual evaluation of 
the cow and the palpation of the udder to detect udder edema caused by overbagging show cows.

The goals of this study were (1) to evaluate the association between various parameters (e.g., milking procedures and treatment data) and the degree of udder edema as determined by sonographic udder edema scoring and (2) to identify risk factors associated with sonographically detectable udder edema in show cows under show conditions. We hypothesized that at shows, PMI and dairy breeds are associated with sonographically detectable udder edema.

Data were collected from 321 cows at 4 highly competitive dairy shows in Switzerland (shows A-D) between January and September 2017. Ten cows were excluded from analyses because of the short interval between calving and sonographic evaluation $(\leq 21 \mathrm{~d}$; physiological postpartum edema possible; Tucker et al., 1992; Waller et al., 2007); finally, data of 311 cows were analyzed. In shows $\mathrm{A}, \mathrm{B}$, and $\mathrm{D}$, exhibitors decided the time of last milking before showing. In show C, the maximal milking interval before showing was given by the show organizers. This was the only show that provided data of official milking times. Data referring to age and breeds of the cows were taken from the show catalogs. The age of the cows (mean \pm SD) was $4.56 \pm 2.20$ yr. The breeds involved were Brown Swiss $(\mathrm{n}=97)$, Holstein-Friesian $(\mathrm{n}=59)$, Jersey $(\mathrm{n}=20)$, Montbéliard $(\mathrm{n}=23)$, Original Brown $(\mathrm{n}=22)$, Red Holstein $(\mathrm{n}=50)$, Simmental $(\mathrm{n}=19)$, and Swiss Fleckvieh $(\mathrm{n}=21)$. Breeds were grouped into dairy breeds (Brown Swiss, Holstein-Friesian, Red Holstein, and Jersey) and dual-purpose breeds (Montbéliard, Original Brown, Simmental, and Swiss Fleckvieh).

Sonographic evaluation of the udders was performed on cows after they were ranked by the judge, in their respective category, and after the grand championships but before milking. Twenty-nine cows were examined twice, and only the second examination was chosen for further analysis. The experimental protocol included the following procedures for each cow: (1) collection of data by interviewing the respective exhibitor, (2) sonographic examination of the 4 quarters to detect and score udder edema according to Balmer et al. (2018), and (3) collection of blood samples to gain serum for laboratory analyses of traces of certain drugs. Results were not given to the owners, and no penalties were given if anomalies were detected.

The DIM were extrapolated from the calving dates provided in the exhibition catalog. Daily milk yields, last milking time, and unequal milking (1 or more quarters at a time different from the others) were collected by asking the exhibitors. The last recorded milk production was obtained by the breeders' association (measured within the past $30 \mathrm{~d}$ before the show). The last milking time was available by the exhibition organizer for show $\mathrm{C}$. The cows were at $115.95 \pm 74.85$ DIM. Anamnestically, the cows yielded $34.32 \pm 8.32$ $\mathrm{kg} / \mathrm{d}$. Based on official milking data of the respective breeders' associations, cows yielded $33.56 \pm 8.01 \mathrm{~kg} / \mathrm{d}$ within the month before evaluation.

Use of superglue or collodion to seal the outer opening of the streak canal was recorded. Information on systemic treatment given during the week preceding the show or at the show or both with NSAID, SAID, or diuretics was retrieved by asking the exhibitors and by obtaining the official veterinary treatment protocols by the organizers. Sedatives are explicitly prohibited at Swiss cow shows (Association of Swiss Cattle Breeders, 2016), and exhibitors were not asked about this. Blood samples were collected from the tail vein for drug detection at the Swiss Laboratory for Doping Analyses (Badoud et al., 2009, 2010). All NSAID, SAID, and diuretics that are currently licensed in Switzerland for use in cattle were tested.

Sonographic examination and scoring was performed according to Balmer et al. (2018). The evaluators were blinded according to the show, the cow, the owner, and the location on the udder. The scores were given by 3 independent observers experienced in reading soft tissue sonographic scans (Balmer, O'Brien, and Steiner). If different scores were obtained, the evaluators rescored the pictures to reach a consensus. The highest score obtained on 1 of the 4 quarters was chosen to determine the score at cow level.

The normality of continuous data was checked with the Shapiro-Wilk test statistic $(>0.95)$. For all parameters of interest, normality was rejected. For analyzing the univariable association between sonographically detectable udder edema score (scores $0,1,2$, and 3) and evaluated parameters, chi-squared (for categorical data) and Kruskal-Wallis 1-way ANOVA on ranks (for continuous data), respectively, were used. A Bonferroni-corrected $P$-value was calculated to account for multiple comparisons; the significance level was set at $P \leq 0.05$ (without Bonferroni adjustment). Wilcoxon rank-sum test was used to compare the milking interval and milk yield between dairy and dual-purpose breeds. Statistical analyses were undertaken using the software package NCSS10 (NCSS LLC, Kaysville, UT; http:/ /www.ncss.com/). For multivariable analyses, sonographically detectable udder edema score was classified in 2 different ways. In model 1, the binary outcome represented 0 (score 0 ) and 1 (scores 1, 2, and 3). In model 2 , the binary outcome represented 0 (scores 0 and 1 ) and 1 (scores 2 and 3). The milking interval was considered to be the biologically most relevant parameter associated with udder edema and was therefore added to the logistic regression models as first parameter. In 
both models, milking intervals were classified into 3 categorical subdivisions $[<14 \mathrm{~h}$ (reference) vs. $\geq 14 \mathrm{~h}$ $<16 \mathrm{~h}$ vs. $\geq 16 \mathrm{~h}$ ]. Additional parameters that were included in univariable screening for association with sonographically detectable udder edema score were shows, dairy versus dual-purpose breeds, unequal milking, use of teat sealing, treatments received before or at the show (information retrieved from the interview with the exhibitors), treatment information retrieved from the veterinary treatment protocols, blood results, cow's age, and DIM. There was no strong correlation among the risk factors ( $\mathrm{r}$ or phi coefficient $<0.7$ ); thus, none of the risk factors had to be excluded from the analysis. The model-building strategy was to initially include individually each parameter with $P<0.25$ in the univariable analysis in the models. This was followed by a stepwise forward selection with elimination of all nonsignificant parameters. Model fit was assessed by the Hosmer and Lemeshow test and a visual assessment of residuals. The sample size of 81 cows in the smallest group (milking interval $<14 \mathrm{~h}$ ) is sufficient to detect a difference of $20 \%$ or more in edema prevalence, assuming a baseline prevalence of $10 \%$ in the unexposed group, a power of $80 \%$, and a significance level of $5 \%$. Sample size calculations were performed with the online calculator WinEpi (http://www.winepi .net). In all descriptive analyses and both models for edema formation, only information retrieved from the interview with the exhibitors concerning milk yield and milking intervals was included because it was available for all shows. To compare the anamnestic with the official data (milk yields and milking intervals), we estimated the correlation coefficient $(\mathrm{r})$. For interpretation, $\mathrm{r} \geq 0.9$ was rated as a very high correlation, $0.68 \leq \mathrm{r}$ $\leq 1.0$ as strong or high, $0.36 \leq \mathrm{r} \leq 0.67$ as modest or moderate, and $\mathrm{r} \leq 0.35$ as low or weak (Taylor, 1990).

The results are presented in Tables 1 and 2. The scores were not evenly distributed when dairy and dual-purpose cows were compared $(P<0.001)$. Milk yield and milking intervals at the respective shows were significantly greater in dairy breeds $(35.61 \pm 7.94 \mathrm{~kg} / \mathrm{d}$; $15.74 \pm 2.67 \mathrm{~h}$ ) than in dual-purpose breeds (30.96 \pm $8.39 \mathrm{~kg} / \mathrm{d} ; 14.26 \pm 2.12 \mathrm{~h}$; both $P<0.001$ ). At show $\mathrm{C}$, milking intervals were significantly lower $(14.59 \mathrm{~h}$ $\pm 1.67 \mathrm{~h})$ compared with the other shows $(15.50 \mathrm{~h} \pm$ $2.75 \mathrm{~h} ; P=0.026)$. Udder edema score was significantly associated with milking intervals $(P<0.001)$ as well as with anamnestic milk yields $(P=0.050)$ and unequal milking $(P=0.014)$. The milking intervals of cows with udder edema scores 2 and 3 were significantly longer compared with udder edema score 0 (Figure 1). Associations between anamnestic milk yields and milk yields based on official milking data of the respective breeder associations and anamnestic and official milking inter- vals at show $\mathrm{C}$ were high $(\mathrm{r}=0.842)$ or moderate $(\mathrm{r}=$ 0.612 ), respectively. Udder edema scores were significantly associated with treatment procedures mentioned in the official treatment protocols $(P=0.031)$. In model 1 , milking intervals of $\geq 16 \mathrm{~h}$ were associated with increased risk for udder edema compared with milking intervals of $<14 \mathrm{~h}$ [regression coefficient $=1.20 ; P=$ 0.008; odds ratio $(\mathrm{OR})=3.33 ; 95 \% \mathrm{CI}=1.37-8.09]$. Show A was associated with increased risk for udder edema compared with show $\mathrm{C}$ (regression coefficient $=1.35 ; P=0.002 ; \mathrm{OR}=3.84 ; 95 \% \mathrm{CI}=1.65-8.97)$. Dairy breeds were associated with increased risk for udder edema compared with dual-purpose breeds (regression coefficient $=2.00 ; P<0.001 ; \mathrm{OR}=7.39 ; 95 \%$ $\mathrm{CI}=2.78-19.69)$. In model 2 , only milking intervals of $\geq 16 \mathrm{~h}$ compared with milking intervals of $<14 \mathrm{~h}$ were associated with increased risk for udder edema (regression coefficient $=2.20 ; P=0.004 ; \mathrm{OR}=9.00,95 \% \mathrm{CI}$ $=2.02-40.09$ ).

Our initial hypotheses that sonographically detectable udder edema is associated with PMI and dairy breeds were confirmed. As dairy breeds were associated with significantly longer milking intervals and sonographically detectable udder edema was not significantly associated with official milk yields, certain preparation practices by the owners (e.g., PMI and unequal milking) seemed to mainly affect sonographically detectable udder edema.

In both of the developed models, it was shown that milking intervals of $\geq 16 \mathrm{~h}$ versus milking intervals of $<14 \mathrm{~h}$ were associated with increased risk of sonographically detectable udder edema. In model 1, comparing the absence of sonographically detectable udder edema to any degree of udder edema, dairy breeds were at higher risk for udder edema compared with dualpurpose breeds. In the same model, it was shown that cows in show A were at higher risk of developing udder edema than cows in show $\mathrm{C}$. Show $\mathrm{C}$ was chosen as a reference because it was the show with the lowest sonographically detectable udder edema prevalence as the show organizers limited maximal milking intervals. The milking interval in show $\mathrm{C}$ was indeed significantly lower than that of the other 3 shows.

Edema occurs in case of inflammation or venous backflow interference or due to compartment differences of oncotic or osmotic pressures. After PMI, a combination of inflammation and venous backflow interference may be responsible for udder edema formation. By using the sonographic udder edema scoring system (Balmer et al., 2018), which was introduced to score udder edema during shows, it is now possible to define risk factors for sonographically detectable udder edema. It seems relevant that this is done in the target population. Highly competitive shows in Switzerland were chosen 
Table 1. Frequency [no. (\%)] of sonographically detectable udder edema (scores 0-3) in different dairy shows (shows A-D); breeds; use of unequal milking at the show; use of teat sealer; treatment with steroidal anti-inflammatory drugs, nonsteroidal anti-inflammatory drugs, and diuretics prior to or at the show (information retrieved from the interviews with the exhibitors); official veterinary treatment protocols; and detection of the previously mentioned drugs in blood

\begin{tabular}{|c|c|c|c|c|c|c|}
\hline \multirow[b]{2}{*}{ Item } & \multicolumn{4}{|c|}{ Maximal sonographically detectable udder edema score } & \multirow[b]{2}{*}{ Total } & \multirow[b]{2}{*}{$P$-value } \\
\hline & 0 & 1 & 2 & 3 & & \\
\hline \multicolumn{7}{|l|}{ Show } \\
\hline A & $134(71.3)$ & $30(16.0)$ & $18(9.6)$ & $6(3.2)$ & 188 & $0.235^{*}$ \\
\hline B & $32(84.2)$ & $5(13.2)$ & $1(2.6)$ & $0(0)$ & 38 & \\
\hline $\mathrm{C}$ & $57(87.7)$ & $6(9.2)$ & $1(1.5)$ & $1(1.5)$ & 65 & \\
\hline $\mathrm{D}$ & $15(79.0)$ & $3(15.8)$ & $1(5.3)$ & $0(0)$ & 19 & \\
\hline Dual purpose & $79(94.1)$ & $3(3.6)$ & $1(1.2)$ & $1(1.2)$ & 84 & \\
\hline \multicolumn{7}{|l|}{ Unequal milking } \\
\hline No & $188(79.0)$ & $32(13.5)$ & $15(6.3)$ & $3(1.3)$ & 238 & 0.014 \\
\hline Yes & $29(67.4)$ & $6(14.0)$ & $4(9.3)$ & $4(9.3)$ & 43 & \\
\hline \multicolumn{7}{|l|}{ Teats sealed } \\
\hline No & $38(88.4)$ & $5(11.6)$ & $0(0)$ & $0(0)$ & 43 & 0.120 \\
\hline Yes & $189(74.7)$ & $36(14.2)$ & $21(8.3)$ & $7(2.8)$ & 253 & \\
\hline Yes & $17(63.0)$ & $5(18.5)$ & $5(18.5)$ & $0(0)$ & 27 & \\
\hline \multicolumn{7}{|c|}{ Treatment protocol $^{3}$} \\
\hline No & $221(77.5)$ & $41(14.4)$ & $16(5.6)$ & $7(2.5)$ & 285 & 0.031 \\
\hline Yes & $17(70.8)$ & $2(8.3)$ & $5(20.8)$ & $0(0)$ & 24 & \\
\hline \multicolumn{7}{|l|}{ Doping findings ${ }^{4}$} \\
\hline No & $116(80.6)$ & $21(14.6)$ & $3(2.1)$ & $4(2.8)$ & 144 & 0.102 \\
\hline Yes & $20(71.4)$ & $5(17.9)$ & $3(10.7)$ & $0(0)$ & 28 & \\
\hline
\end{tabular}

${ }^{1}$ Systemic anamnestic treatment with steroidal or nonsteroidal anti-inflammatory drugs or diuretics or both during the last $7 \mathrm{~d}$ before the show. ${ }^{2}$ Systemic anamnestic treatment with steroidal or nonsteroidal anti-inflammatory drugs or diuretics or both during the stay on the showground. ${ }^{3}$ Systemic treatments with steroidal or nonsteroidal anti-inflammatory drugs or diuretics or both during the stay on the showground based on analyses of official treatment protocols.

${ }^{4}$ Results based on laboratory analyses of blood samples. The analyses contained steroidal and nonsteroidal anti-inflammatory drugs and diuretics.

*If sonographically detectable, udder edema scores were classified as 0 (score 0) or 1 (scores 1,2 , and 3 ) and compared with the 4 shows, it resulted in significantly different distribution $(P=0.033)$.

for the current study; therefore, the Swiss show cow population is well represented. The methodology might be adaptable to other countries, considering that some parameters may differ.

Anamnestic information was used for the analyses because it represented a uniform and complete data set. Milk yields from the Swiss cows of all 4 shows and milking intervals from 1 show were available as both anamnestic and official data. Thus, correlation estimation to check reliability of anamnestic data was possible. Correlation was high for milk yield and moderate for milking intervals. For the latter parameter, official data $(\mathrm{n}=46)$ from only 1 show were available, making the correlation results only moderately reliable. Nevertheless, we conclude that the reliability of anamnestic data is moderate to good. However, anamnestic data do not represent hard data. Because we do not have more hard data to check the reliability and honesty of the given information, the use of anamnestic data represents a limitation of our study. In the vast majority of national and international shows, the real milking intervals are not known because control of milking time by the organizers or veterinary officials is not possible. Therefore, the use of hard data was impeded by the circumstances given by the traditional show rules. Data from official treatment protocols included only treatments initiated by the responsible show veterinarians after clinical examination (other treatments are not allowed in cows at Swiss cow shows; Association of Swiss Cattle Breeders, 2016). If other treatments still had occurred, they were not recorded. Results from laboratory analyses have their well-described limits (Badoud et al., 2009, 2010) but grant high sensitivity and specificity.

Sonographically detectable udder edema was significantly associated with dairy breeds, PMI, treatments according to official treatment protocols, and unequal milking but not with high milk yields. It seems that dairy breeds were not affected more frequently by ud- 


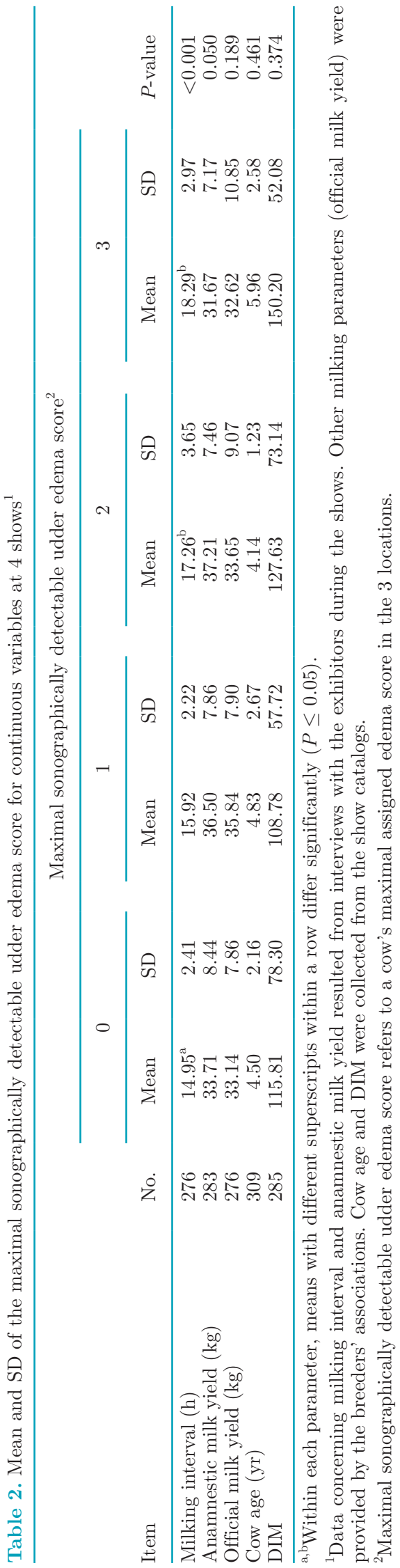

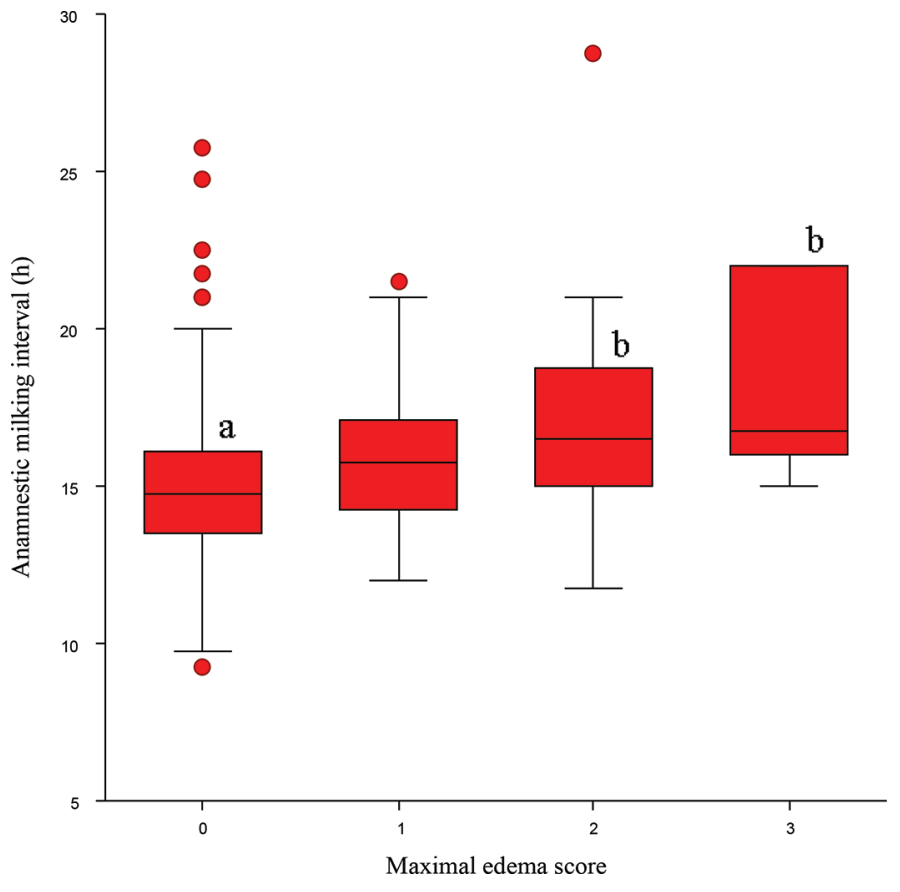

Figure 1. Box plot representing the comparison of anamnestic milking intervals $(\mathrm{h})$ with sonographic udder edema score. Anamnestic milking intervals that are significantly different among maximal edema scores are marked with different letters $(a, b)$.

der edema because of the greater milk yields but rather because owners of these breeds were more inclined to manipulate their cows (e.g., unequal milking) and further extend the milking interval during show preparation compared with owners of dual-purpose breed cows due to higher importance of the udder in dairy breeds. Because correlation with milking interval was high, udder edema seems to represent a pathological sign. Kohler et al. (2016) demonstrated the negative effect on a cow's health and behavior after a prolonged (24-h) milking interval. Therefore, overbagging represents a remarkable welfare issue.

In both statistical models, milking intervals represented the most important risk factor for sonographically detectable udder edema. Therefore, testing for and grading of sonographically detectable udder edema seems to be a suitable tool for objectively identifying and classifying cows with overbagged udders during cow shows. As edema formation is a sign of a nonphysiological status, a cut-off between scores 0 and 1 is justified. Milking intervals represent significant risk factors in both models ( $<14 \mathrm{~h}$ vs. $\geq 16 \mathrm{~h}$ ), but OR differ markedly. In model 2 , OR is at 9.00 , which represents a very strong risk factor (compared with $\mathrm{OR}=3.33$ in model 1). This could favor a cut-off between scores 1 and 2 . 
Only the milking interval represented a relevant risk factor in both statistical models of this study; therefore, it can be concluded that the risk for the presence of sonographically detectable overbagging edema is mainly associated with PMI. Sonographic examination is a feasible technique for detecting overbagging under show conditions, and it may accordingly support the improvement of animal welfare at dairy shows.

\section{ACKNOWLEDGMENTS}

This study was generously supported by the Swiss Federal Food Safety and Veterinary Office (Bern, Switzerland) and the Association of Swiss Cattle Breeders (Zollikofen, Switzerland). Henry Schein Animal Health (Lyssach, Switzerland) generously provided the scanner for the study. We thank the students of veterinary medicine, Vetsuisse-Faculty, University of Bern and involved veterinarians for their help in collecting the data. We are grateful to show officials and show cow owners for their patience during data collection.

\section{REFERENCES}

Association of Swiss Cattle Breeders. 2016. Show regulations pertaining to the preparation and presentation of animals at dairy cattle shows in Switzerland. Accessed Oct. 2018. https://asr-ch.ch/wp -content/uploads/2017/02/1100.04_ASR-Show-Regulations_2016 $-12-22$.pdf.

Badoud, F., E. Grata, L. Perrenoud, L. Avois, M. Saugy, S. Rudaz, and J. L. Veuthey. 2009. Fast analysis of doping agents in urine by ultra-high-pressure liquid chromatography-quadrupole time-offlight mass spectrometry I. Screening analysis. J. Chromatogr. A 1216:4423-4433.

Badoud, F., E. Grata, L. Perrenoud, M. Saugy, S. Rudaz, and J. L. Veuthey. 2010. Fast analysis of doping agents in urine by ultrahigh-pressure liquid chromatography-quadrupole time-of-flight mass spectrometry. II: Confirmatory analysis. J. Chromatogr. A 1217:4109-4119.

Balmer, M., M. Alsaaod, M. Boesiger, R. O'Brien, and A. Steiner. 2018. Technical note: Evaluation of a sonographic overbagging edema scoring system for show cows: Comparison with visual inspection. J. Dairy Sci. 101:7494-7499.

Kohler, P., M. Alsaaod, G. Dolf, R. O'Brien, G. Beer, and A. Steiner. 2016. A single prolonged milking interval of $24 \mathrm{~h}$ compromises the well-being and health of dairy Holstein cows. J. Dairy Sci. 99:9080-9093.

O'Brien, R. T. 2017. Over bagging in dairy show cows: An ethical crisis. J. Am. Vet. Med. Assoc. 251:271-272.

Taylor, R. 1990. Interpretation of the correlation coefficient: A basic review. J. Diagn. Med. Sonogr. 6:35-39.

Tucker, W. B., G. D. Adams, M. Lema, M. Aslam, I. S. Shin, P. Leruyet, and D. L. Weeks. 1992. Evaluation of a system for rating edema in dairy cattle. J. Dairy Sci. 75:2382-2387.

Waller, K. R., R. T. O'Brien, and S. M. McGuirk. 2007. Ultrasonographic distribution and duration of udder edema in post-partum and over-bagged dairy cows. Bov. Pract. 41:129-133. 